\title{
Proceeding Paper \\ Going Underground for Ferronickel Mining in Greece: Preliminary Feasibility and Potential Benefits ${ }^{\dagger}$
}

\author{
Andreas Benardos ${ }^{1, *(\mathbb{D}}$, Ioannis Vlachogiannis ${ }^{2}{ }^{(1)}$, Christos Stenos $^{3}$, Spyros Pappas ${ }^{3}$, Georgios Bourmas ${ }^{4}$, \\ Sotirios Koukoumtzis ${ }^{3}$ and Nikolaos Koronakis ${ }^{5}$ \\ 1 School of Mining \& Metallurgical Engineering, NTUA, 15780 Athens, Greece \\ 2 Geomine J\&K, Metamorfosi, Karystou 34, 14451 Athens, Greece; i.m.vlachogiannis@gmail.com \\ 3 LARCO GMMSA, 27 Fragkokklisias St Maroussi, 15125 Athens, Attica, Greece; christos.stenos@larco.gr (C.S.); \\ spyros.pappas@larco.gr (S.P.); sotiris.koukoumtzis@larco.gr (S.K.) \\ 4 Grecian Magnesite SA, 63100 Yerakini, Greece; g.bourmas@grecianmagnesite.com \\ 5 Omikron Kappa Consulting SA, 15122 Maroussi, Greece; n.koronakis@omikronkappa.gr \\ * Correspondence: abenardos@metal.ntua.gr; Tel.: +30-2107722182 \\ + Presented at International Conference on Raw Materials and Circular Economy, Athens, Greece, \\ 5-9 September 2021.
}

check for updates

Citation: Benardos, A.;

Vlachogiannis, I.; Stenos, C.; Pappas,

S.; Bourmas, G.; Koukoumtzis, S.; Koronakis, N. Going Underground for Ferronickel Mining in Greece:

Preliminary Feasibility and Potential Benefits. Mater. Proc. 2021, 5, 16.

https://doi.org/10.3390/

materproc2021005016

Academic Editor: Anthimos Xenidis

Published: 16 November 2021

Publisher's Note: MDPI stays neutral with regard to jurisdictional claims in published maps and institutional affiliations.

Copyright: (c) 2021 by the authors. Licensee MDPI, Basel, Switzerland. This article is an open access article distributed under the terms and conditions of the Creative Commons Attribution (CC BY) license (https:// creativecommons.org/licenses/by/ $4.0 /)$.

\begin{abstract}
The gradual elimination of rich and surficial-located Ni reserves mandates the assessment regarding the gradual switch from open-pit mines to underground mining schemes. This could allow for the continuation of the steady ore supply and furthermore could assist in minimizing the environmental footprint of the exploitations. This paper investigates the possibility of adopting an underground exploitation scheme and provides data on the preliminary feasibility of the endeavor for the Vrysakia deposit that was selected as a model project. It was found that such solutions proved to be technically sound, also yielding considerable results from a financial viewpoint.
\end{abstract}

Keywords: pre-feasibility assessment; underground mine design; ferronickel deposits; open pit to underground transition

\section{Introduction}

Nickel has always been an important metal used in metallurgy, primarily for the development of stainless steel, which accounts for more than $70 \%$ of its annual consumption [1]. Over recent years, Ni's contribution to the development of batteries, especially in the automotive industry, has been discussed extensively, raising issues about the adequacy of the global and EU Ni supply [2]. Currently (2020), Ni supply from mines is around $2500 \mathrm{ktpa}$, while over the next decade this supply is expected to rise further at a CARG of $4.7 \%[3]$.

Greece has always had a dominant place in European nickel production and historically has been the greatest producer in the EU; since 2017, it has fallen to 2nd place, behind Finland [4]. Greek ferronickel (Fe-Ni) alloy production is at around 20.000 tpa, with LARCO GMMSA representing the sole nickel miner and producer in the country. The ferronickel lateritic deposits located in the central Evia, in Ag. Ioannis and Kastoria are significant, summing a total annual ore production of approx. $1.3 \mathrm{Mt}$. The systematic exploitation of ferronickel deposits in Greece goes back to the 1950s, where in the area of Agios Ioannis, small surface mines began to develop followed by the first large scale underground nickel mine in that area [5]. Nevertheless, this exploitation period of more than 50 years has now led to the gradual depletion of the richer and more surficial and "easy" to exploit deposits. In Figure 1 the average Fe-Ni ore yearly production is given, along with the average quality ( $\mathrm{Ni} \%$ ) attained. As depicted, production is relatively stable, while quality shows a gradual deterioration over the years, being now just below $1 \%$.

The ferronickel deposits are still available for exploitation, but they are gradually sinking towards deeper horizons, experiencing a gradual increase in stripping ratios, and 
leading to higher production costs and more challenges in terms of waste management. Surface mining activities will continue without experiencing any problems, yet in a pursuit to address the new challenging conditions over a medium to long-term horizon, careful planning and exploration of further options should be commenced at this stage.

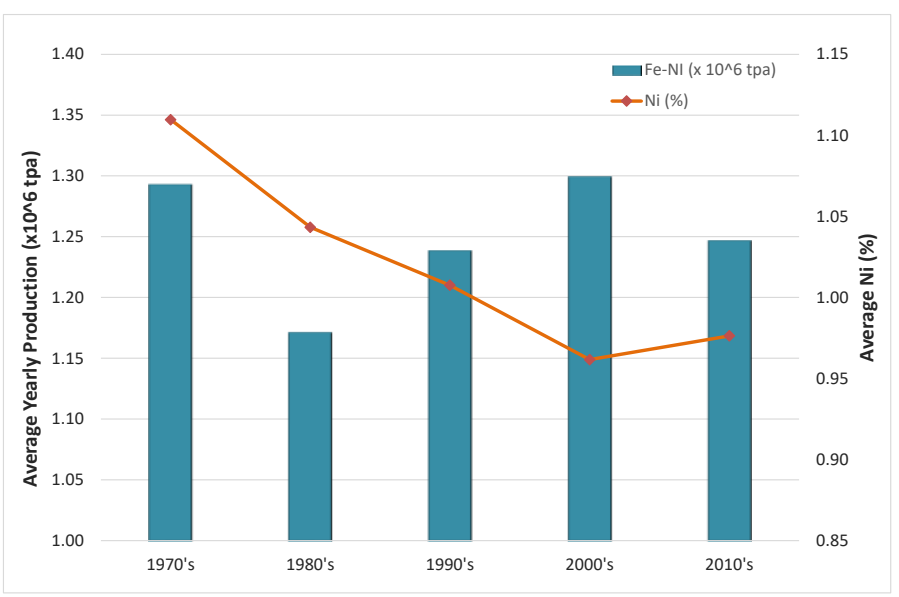

Figure 1. LARCO GMMSA production and Ni content over a 50-year period.

A radical solution to this could be represented by the implementation of underground mining schemes, which can overall improve the production characteristics of the company's ore deposits. This can, first of all, lead to a drastic reduction in waste production and minimize problems associated with surface disposal areas, as well as their aftercare. At the same time, it will allow for the exploitation of deposits, or to reach rich zone areas, that up to now could not been mined at competitive-at least in relation to today'sexploitation costs. Therefore, with the adoption of such a strategic move, the company can achieve improved production costs, drastically reduce its environmental footprint and also raise a significant increase in its usable reserves, ultimately leading to an increase in its direct assets.

This concept for the gradual transition from surface to underground operations is not a new one and several researchers have investigated $[6,7]$ or documented such decisions taken in the mining industry [8]. Of course, such a strategic leap forward requires a wellorganized investigation and evaluation of all parameters, as well as the proper preparation for this drastic change in the operation process-especially in cases like LARCO GMMSA, where mining companies are exclusively focusing on surface mining and lack essential know-how of underground mining operations. In assessing this option, certain issues should be dealt with in order to obtain a more straightforward and realistic approach to the true potential of underground mining schemes. This paper presents the main findings of the research undertaken to provide such initial answers on the potential of developing underground ferronickel exploitations in Greece, to assess what is required in terms of resources and evaluate their overall feasibility.

\section{The Vrysakia Deposit}

LARCO GMMSA has more than a dozen orebodies in the Evia region that are currently performing surface exploitation. For the possible transition from surface to underground mining, the main strategy selected was to identify the most promising orebody and to evaluate its potential as an underground exploitation scheme. This evaluation, upon a positive result, could actually serve as the onset—and as a basic pilot guide-for the subsequent transformation of the company from an open-pit operator to an underground mining producer. The methodology that was followed has been divided into two main identification and selection phases-the first focusing on the screening of all available deposits and the shortlisting of the most important ones, while the second included the actual analysis and pre-feasibility assessment of this target deposit. 
The above process identified the Vrysakia deposit as the most promising one. The section under investigation for the development of the underground exploitation scheme has a length of about $1.3 \mathrm{~km}(0.8 \mathrm{~km}$ to $1.8 \mathrm{~km})$ with a NW-SE direction. It is located between the Cretaceous and Jurassic limestones of the HW and FW, respectively, and dips at an angle of $45^{\circ}$ to SW, extending below the current pit limit (Figure 2a). It reaches a maximum depth of $250 \mathrm{~m}$, whereas its central area is located almost $150 \mathrm{~m}$ below the surface. The orebody thickness ranges from 10 to $30 \mathrm{~m}$ and the $\mathrm{Ni}(\%)$ content ranges from $0.55 \%$ to $1.15 \%$ (Figure $2 b$ ), with an average value of $0.95 \%$. At a cut-off grade of $0.8 \% \mathrm{Ni}$, this ensures the maximization of the reserves; the Vrysakia deposit's reserves are estimated at around $15 \mathrm{Mt}$.

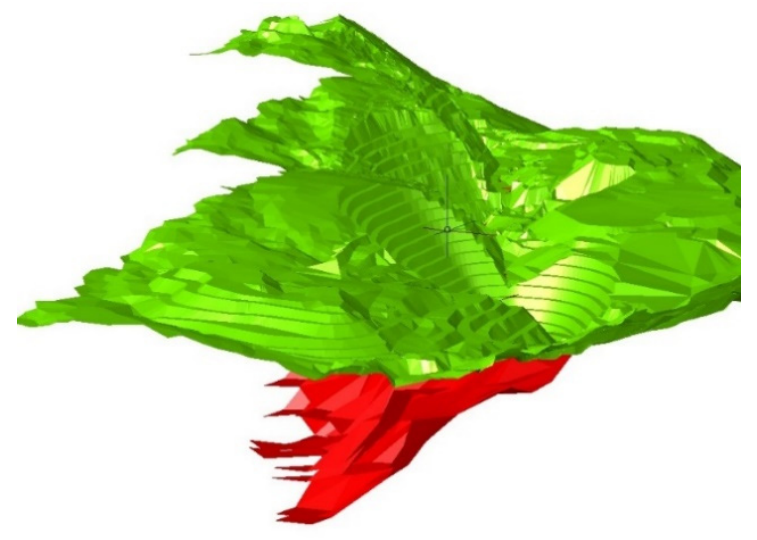

(a)

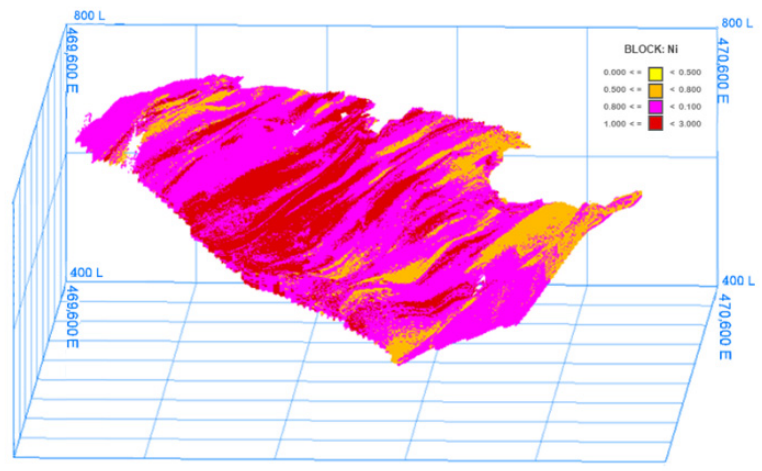

(b)

Figure 2. Vrysakia deposit: (a) General layout of the existing mine and the deposit; (b) Block model of the Ni (\%) content.

A geotechnical investigation campaign was carried out [9], indicating that the orebody primarily, and the surrounding formations, are characterized by relatively low to medium strength as well as low to medium quality in terms of GSI classification ( $\mathrm{c}_{\text {ore }}: 250-350 \mathrm{kPa}$, phi ore: $23-28^{\circ}$, GSI $\left.I_{\text {ore }}: 20-45\right)$.

\section{Pre-Feasibility Assessment}

The main phase of the analysis is the part where major decisions are to be taken for the underground mining scheme, so as to measure the impacts attained in terms of production and mining costs. The pre-feasibility assessment is aimed primarily at assessing whether there is merit in pursuing the solution examined and provides basic data that can assist in the go/no-go decision, that needs to be taken. Of course, such guidelines need further elaboration in order to further narrow down uncertain issues and provide a more accurate cost assessment of the capital expenditure and operational costs achieved, as this stage offers estimates that are within $20-30 \%$ accuracy [10].

\subsection{Mining Method Assessment and Selection-Design and Technical Issues}

The selection of the mining method is probably one of the principal decisions that should be taken for design purposes. This dictates the whole operation of the mining scheme and sets special provisions and requirements for the accompanying underground development works, governs the operation characteristics, machinery types and fleets required, and, thus, the overall cost figures for the exploitation scheme.

In the Vrysakia deposit, the low quality of the geotechnical setting indicates that the principal categories of caving and filling methods can be prioritized over the open stoping ones. The UBC mining selection method [11] was employed, which highlighted the methods of (i), overhand cut-and-fill, (ii) room-and-pillar with backfilling (post-pillar mining) and (iii) sub-level caving as the more promising options for the development of the underground mining scheme. 
Each of these methods showed pros and cons. The cut-and-fill is relatively straightforward, offering at the same time maximum protection to the workers and against surface subsidence issues; the post-pillar is widely known in Greece and is easily adaptable, while the caving offers financial benefits. These three mining schemes were further elaborated in an attempt to firstly assess the overall stability of the mine and thus optimize the proposed dimensioning of all workings, and secondly to determine the operation cost for each scheme. In this context, the mining methods under investigation were assessed by their performance in delivering the anticipated results in terms of safety, production and cost [9], having a predetermined annual production target of $350,000 \mathrm{t}$, so as to set a common analysis framework that every underground mining scheme should comply with. Given the recovery and dilution rates attained in each scheme, the overall mine life ranged from 30 to 38 years. The cut-and-fill had the more extended mine life, as it surpassed the recovery ratio of the two others, having a total production of approx. $13.5 \mathrm{Mt}$.

In all schemes, the orebody is reached through two (2) main access ramps, approx. $4 \mathrm{~km}$ in total length, by adopting a typical $5 \mathrm{~m} \times 5 \mathrm{~m}$ horseshoe cross-section. As the orebody can be easily accessible right away from the existing pit limit (besides the crownpillar left in place), these tunnels actually follow the orebody's extension and development towards the lower horizons, down to a maximum depth of $250 \mathrm{~m}$. This forms the key infrastructure that facilitates easy access to every part of the deposit and forms the ventilation network. Through these ramps, the development works commence so as to reach and intersect the orebody as well as to form the production drifts $(4.5 \mathrm{~m} \times 5 \mathrm{~m})$ that constitute the main exploitation levels, as, for example, is shown in Figure 3 for the case of the cut-and-fill mining method.

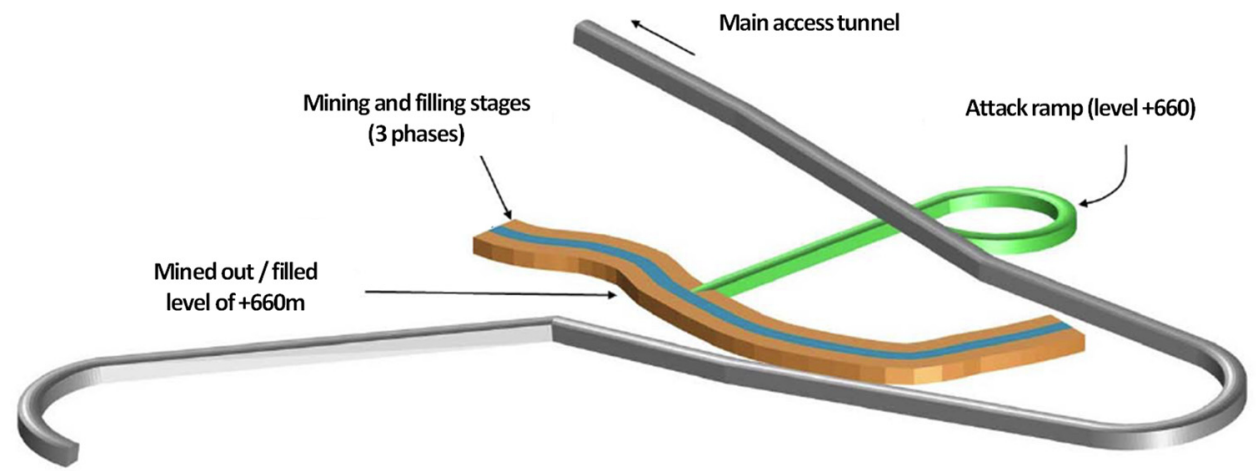

Figure 3. Development of the cut-and-fill mining scheme at the $+660 \mathrm{~m}$ level [9].

\subsection{Cost and Financial Assessement}

\subsubsection{Estimation of the Capital (CAPEX) and Operating Expenditure (OPEX)}

In order to estimate the CAPEX, the required infrastructure, development works and machine fleet need to be taken into account. The most critical parameter lies in the total length of the access tunnels and drifts required in order to facilitate ore production. This will ultimately determine the capital cost as well as the duration of preparatory works before the commencing of ore production.

Based on the preliminary mine design, the total length of these tunnels was estimated in the order of $4 \mathrm{~km}$ for the cases of cut-and-fill and room-and-pillar mining methods. For the case of sublevel caving mining, this length can be further increased if the production drifts are to be considered and included in the CAPEX. Obviously, the total length of the access works required for sublevel caving should by no means burden the CAPEX only. This network of access works will have to be formed eventually, but since this task is envisaged to be carried out concurrently with the operational phase, the related costs were evenly distributed and taken into consideration in a staged manner throughout the mine-life. Thus, the total CAPEX needed for the caving method was split in two fractions, i.e., one part that burdens the CAPEX, focusing only on the drifts rendered necessary for 
the initiation of ore production, and taking into account the fact that the mining is first started from the top and then progresses to the lower parts of the deposit; while the second part, including all the others, was moved to OPEX. This can be seen in Figure 4a, where the initial EUR 17 million of CAPEX were adjusted to approx. EUR 6 million, while the excess amount was transferred and allocated to the yearly OPEXs.

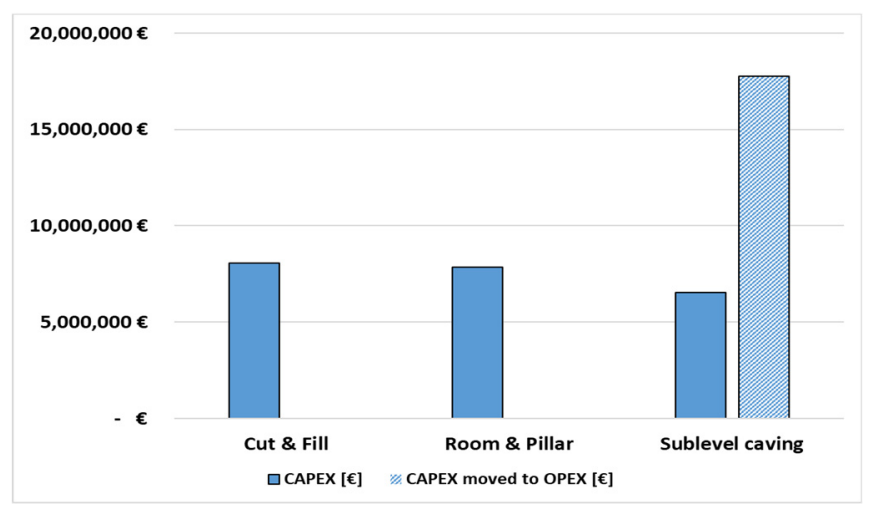

(a)

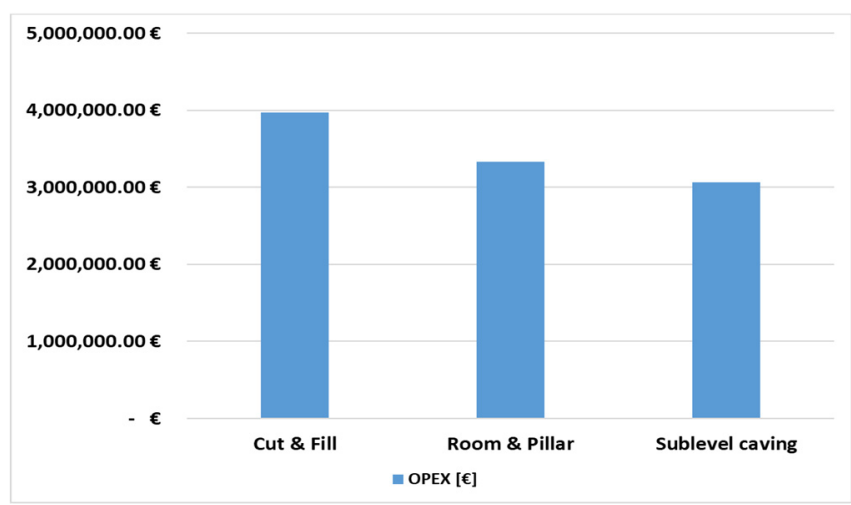

(b)

Figure 4. (a) CAPEX and (b) Yearly OPEX estimation for each of the underground mining scenarios.

Given the characteristics and parameters of the operation under each scheme, the procedure, sequencing and resources required, and their respective costs, have been used to assess the daily, monthly and yearly operating expenditures. As expected, the most demanding operation in terms of operating cost is the cut-and-fill method, as additional resources for the backfilling preparation and deployment phase are required. In terms of yearly operating expenses, the costs for each scenario are presented in Figure $4 \mathrm{~b}$.

\subsubsection{Financial Assessment of the Underground Mining Project}

The assessment of the project is conducted using the net present value (NPV) criterion. Under this context, all CAPEX and OPEX data, as well as estimated revenues, under all mining scenarios have been estimated using the discounted cash flow (DCF) valuation method. This has been done for the whole life span of the project using a discount rate of $8 \%$. A typical selling price of the $\mathrm{Ni}-\mathrm{Fe}$ ore coming from the mine (run-of-mine-ROM), was also adopted to estimate the proceedings of the mining. Under this concept, the ore is produced at the mine and sold directly to LARCO GMMSA. Hence, the Vrysakia underground exploitation can be seen as a discrete mining unit. The estimation of the selling price of the ore has been made using individual characteristics in terms of Ni content $(\%)$ and moisture (\%), as well as based on an anticipated Ni LME trade price for the coming years, taken at EUR 12,430/t. Accordingly, the ROM price of the ore was EUR 20/t.

The results obtained for the NPV of the three mining schemes are given in Table 1. As depicted, it can be seen that each solution yields considerable positive results. The sensitivity performed addressing potential LME price fluctuations indicated a $15-25 \%$ price margin until the project's break-even point could be reached.

Table 1. NPV assessment for each one of the analyzed underground mining scenarios.

\begin{tabular}{cc}
\hline Underground Mining Method & NPV $[\times \mathbf{1 0 0 0} €]$ \\
\hline Overhand Cut \& Fill & 17,650 \\
Room \& Pillars with backfilling (overhand) & 21,350 \\
Sublevel Caving & 14,550 \\
\hline
\end{tabular}




\section{Conclusions}

The underground exploitation of the ferronickel deposits of LARCO GMMSA is something that undoubtedly will take place in the medium to long term future. This development requires a proper assessment that will safeguard that the transition from the ongoing surface mining approach to an underground one will be made in a smooth and productive manner, that will eventually benefit the company and all stakeholders involved. The analysis presented in this paper offers the first tangible data in terms of what can be achieved. There are several cases that are of interest and can be exploited by underground methods, while the first financial figures show quite encouraging results, indicating a first green light for this development. The mining methods analyzed can get the work done and present special qualities and challenges that need to be taken into account. All of the above examined underground mining methods provided positive NPV values and seem to ensure the feasibility of an underground mining scheme. Nevertheless, these initial data have made it clear that the mining methods of room-and-pillar and cut-and-fill seem to be the leading candidates for further implementation. The caving method seems the weakest among the three, not only in terms of financial performance, but also in terms of potential surface disturbance and environmental issues.

Having said the above, it seems that the cut-and-fill mining method ensures the maximization of ferronickel deposit recovery. The very nature of its principles and operation characteristics facilitates the development of a modern mining scheme, guaranteeing the safety of the underground working conditions and a minimal surface disturbance. Furthermore, it can allow for the proper disposal of waste materials, resulting from other LARCO's mining operations, that could be utilized for filling operations-minimizing the overall environmental footprint of LARCO's exploitations in the region. In this manner, the cut-and-fill mining scenario exhibits the strongest potential both in terms of feasibility and environmental protection, and should be prioritized over the others.

Of course, even if the development of such an underground mining approach is obviously positive, a carefully designed roadmap is undeniably needed. Through this, the accuracy of these first analyses can be further improved and, at the same time, the foundations for the selection of specific steps that need to be taken can be laid out so as to facilitate the implementation of the underground exploitation's design.

Funding: The work presented in the paper has been funded by LARCO GMMSA.

Informed Consent Statement: Not applicable.

\section{References}

1. Nickel Institute. Nickel in the European Union. 2012. Available online: https://www.oma.on.ca/en/multimedialibrary/ resources/NickelintheEuropeanUnionPDF.pdf (accessed on 8 March 2021).

2. Fraser, J.; Anderson, J.; Lazuen, J.; Lu, Y.; Heathman, O.; Brewster, N.; Bedder, J.; Masson, O. Study on Future Demand and Supply Security of Nickel for Electric Vehicle Batteries; Publications Office of the European Union: Luxembourg, 2021.

3. Roskill. Nickel—Outlook to 2030, 17th ed.; Roskill: London, UK, 2020.

4. Euromines. Mining in Europe: Production by Mineral; Euromines: Brussels, Belgium, 2020. Available online: http://www. euromines.org/mining-europe/production-mineral\#Nickel (accessed on 8 March 2021).

5. Benardos, A. Key Aspects of Underground Nickel Mining in Greece. In Proceedings of the Mine Planning and Equipment Selection Symposium-MPES 2007, Bangkok, Thailand, 11-13 December 2007; pp. 80-87.

6. Bakhtavar, E.; Shahriar, K.; Oraee, K. Transition from Open-Pit to Underground as a New Optimization Challenge in Mining Engineering. J. Min. Sci. 2009, 45, 485-494. [CrossRef]

7. Chung, J.; Topal, E.; Ghosh, A.K. Where to make the transition from open-pit to underground? Using integer programming. J. S. Afr. Inst. Min. Metall. 2016, 116, 801-808. [CrossRef]

8. Fiscor, S. Major Open-Pit Copper Mines Move Underground. Eng. Min. J. 2010. Available online: https://www.e-mj.com/ features / major-open-pit-copper-mines-move-underground (accessed on 8 March 2021).

9. Rumbu, R. Introduction to Mining Business Projects; Createspace Independent Publishing Platform: Lolina, SC, USA, 2017.

10. Omikron Kappa Consulting SA. Prefeasibility Assessment Regarding the Underground Nickel Ore Exploitations of LARCO GMMSA in Evia; Report; Omikron Kappa Consulting SA: Athens, Greece, 2019.

11. Miller-Tait, L.; Pakalnis, R.; Poulin, R. UBC Mining Method Selection. In Proceedings of the Mine Planning and Equipment Selection Symposium-MPES 1995, Calgary, AB, Canada, 31 October-3 November 1995; pp. 163-168. 